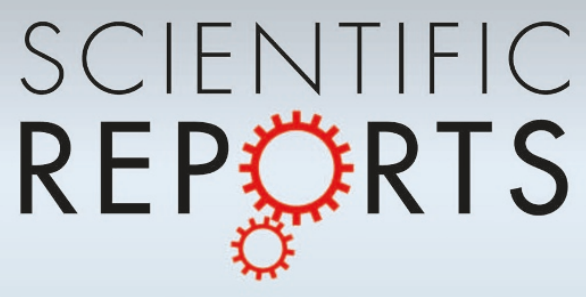

\section{OPEN}

SUBJECT AREAS:

PARASITIC INFECTION

POLICY AND PUBLIC HEALTH

Received

10 October 2013

Accepted

29 January 2014

Published

14 February 2014

Correspondence and requests for materials should be addressed to N.M. (tsatab@lycos. com)

\title{
Soil-Transmitted Helminth Infections and
} Associated Risk Factors in Three Orang Asli Tribes in Peninsular Malaysia

\author{
Tengku Shahrul Anuar' ${ }^{1}$ Fatmah Md Salleh² \& Norhayati Moktar ${ }^{2}$
}

'Department of Medical Laboratory Technology, Faculty of Health Sciences, Universiti Teknologi MARA, Puncak Alam Campus, 42300 Selangor, Malaysia, ${ }^{2}$ Department of Parasitology and Medical Entomology, Faculty of Medicine, Universiti Kebangsaan Malaysia, Jalan Raja Muda Abdul Aziz, 50300 Kuala Lumpur, Malaysia.

Currently, information on prevalence of soil-transmitted helminth (STH) infections among different tribes of Orang Asli (aboriginal) is scarce in Malaysia. The present study is a cross-sectional study aimed at determining the factors associated with the prevalence of STH infections among the Proto-Malay, Negrito and Senoi tribes. Faecal samples were collected from 500 participants and socioeconomic data was collected via pre-tested questionnaire. All samples were processed using formalin-ether sedimentation and Wheatley's trichrome staining. Trichuris trichiura (57\%) was the most common STH seen among the participants, followed by Ascaris lumbricoides $(23.8 \%)$ and hookworm (7.4\%). Trichuriasis and ascariasis showed an age-dependency relationship; significantly higher rates were observed among Senois who aged $<\mathbf{1 5}$ years. Likewise, Negritos also showed an age-dependency association with ascariasis affecting mainly the under 15 years old individuals. Multivariate logistic regression model indicated the following predictors of trichuriasis among these communities; being aged $<15$ years, consuming raw vegetables, belonging to a large household members $(\geq 8)$ and earning low household income $(<$ RM500). Meanwhile, ascariasis was significantly related to participants being aged $<15$ years and earning low household income. Two risk factors were found to be associated with hookworm infection; consuming raw vegetables and eating contaminated fresh fruits.

nfections with soil-transmitted helminths (STHs) affect more than one billion people, particularly the rural poor of the developing world ${ }^{1}$. Approximately one third of the world's population is infected with at least one species of STH, with Ascaris lumbricoides infecting 800 million people, Trichuris trichiura 600 million, hookworm 600 million and resulting in up to 135,000 deaths annually ${ }^{2}$. STHs have also been classified among the most prevalent neglected tropical diseases as they persist exclusively in the poorest populations (i.e., $<$ US $\$ 1.25$ per day) and thus are often forgotten ${ }^{3}$.

Warm climates and adequate moisture are essential for the hatching or embryonation of STH eggs in the environment or development of larvae. Important contextual determinants for human infection are poverty, lack of sanitation and inadequate hygiene (i.e., absence of hand washing with soap after defecation and before eating and walking barefoot) ${ }^{4,5}$. In such social-ecological systems, multiple species of STH infections are common ${ }^{6}$. Transmission of STHs occurs via contact with contaminated soil (hookworm) or consumption of egg-contaminated foods (A. lumbricoides and T. trichiura $)^{4}$. An important epidemiological feature is their highly aggregated distribution; the majority of patients harbour low intensity infections, while only few individuals harbour very heavy infections ${ }^{7}$. People infected with STHs may suffer from anaemia, growth stunting, diminished physical fitness and impaired cognitive development $t^{6}$, representing a persistent drain on socioeconomic development of low-income countries, ${ }^{8,9}$.

In Malaysia, STHs are considered as endemic infections and the prevalence rates vary considerably across different areas (populations). The infections are predominantly seen in children, especially in the underprivileged communities ${ }^{10-13}$. Although the incidence of clinical consequences associated with these infections is very low, they are still a matter of public health concern in Malaysia because the prevalence and the intensity of these infections are still high. Furthermore, the prevalence of STHs in Malaysia is worse in low-income communities which include Orang Asli (aboriginal) group $s^{10,12,14}$, rural poor Malays ${ }^{15}$, children in estates ${ }^{16}$ and squatter $\operatorname{areas}^{17-19}$. 
Within this context, we conducted a cross-sectional study to provide a comprehensive data of the current status and factors associated with the high prevalence of STHs among different Orang Asli tribes in Peninsular Malaysia. The establishment of such data will be beneficial for the public health authorities to reassess the effectiveness of the current control programme (i.e., deworming programme every six months and the usage of a broad spectrum anthelminthics such as albendazole and mebendazole) and planning of more specific control strategies and policies in combating the infections among the ProtoMalay, Negrito and Senoi tribes.

\section{Results}

Study cohort and socioeconomic profile. From 795 enrolled participants, $31.8 \%$ failed to submit their faecal samples and/or were absent during parasitological survey. Thirteen participants had no PVA-fixed faecal sample and 29 participants were absent during the household-based interviews and hence their socioeconomic status could not be determined. Overall, a total of 500 participants (62.9\%) were present during the cross-sectional study and respond to the questionnaire. Among this cohort, 150 individuals (30\%) were from the Proto-Malay tribe, 139 (27.8\%) from the Negrito tribe, and $211(42.2 \%)$ from the Senoi tribe. Proto-Malays comprised of 66 males (44\%) and 84 females (56\%) aged between 2 and 70 years old with a median age of 24 years [interquartile range (IQR) 1039]. One hundred and thirty nine participants from Negritos [66 males (47.5\%); 73 females (52.5\%)] aged between 2 and 74 years old with a median age of 13 years [interquartile range (IQR) 7-30], and 211 respondents among Senois [87 males (41.2\%); 124 females $(58.8 \%)$ ] aged between 2 and 70 years old with a median age of 20 years [interquartile range (IQR) 10-34] participated in this study. General characteristics of each tribe, including their socioeconomic profile are presented in Table 1.

Prevalence of soil-transmitted helminth infections. Trichuriasis was the highest infection found among Negritos (81.3\%), Senois (49.8\%) and Proto-Malays (44.7\%) as compared to ascariasis and hookworm infection (Table 2). A. lumbricoides and T. trichiura showed significantly higher infections in the Senoi (A. lumbricoides: $P<0.001$; T. trichiura: $P=0.023$ ) and Negrito (A. lumbricoides: $P=0.001)$ tribes among those aged $<15$ years. T. trichiura infection was significantly high in Negritos as compared to ProtoMalays (Negrito versus Proto-Malay: $\chi^{2}=39.864 ; P<0.001$ ) and Senois (Negrito versus Senoi: $\chi^{2}=35.468 ; P<0.001$ ). In contrast, $A$. lumbricoides infection was significantly high in Senois as compared to Proto-Malays (Senoi versus Proto-Malay: $\chi^{2}=60.868 ; P<0.001$ ) and Negritos (Senoi versus Negrito: $\chi^{2}=22.225 ; P<0.001$ ). Although the prevalence rate of hookworm infection was high in Negrito (10.1\%), but the difference was not statistically significant between the tribes. No significant difference of STH infections between genders in all tribes studied was observed.

Parasite associations. Table 3 summarize significant associations between T. trichiura, A. lumbricoides and hookworm infections with the demographic and lifestyle factors among different Orang Asli tribes. As for the Negrito tribe, four variables were found to be significantly associated with the T. trichiura infection; being aged less than 15 years, bathing and washing in the river, low father's education and earning low household income. Moreover, univariate analysis identified only one factor that significantly associated with the infection among Senois which is being aged less than 15 years. Among the Proto-Malay tribe, indiscriminate defecation, consuming raw vegetables, low mother's education, belonging to a large household members and low household income were found to be positively significantly associated with trichuriasis.

One variable was found to be significantly associated with ascariasis among the Negrito and Senoi tribes which was being aged $<15$ years. Among the Proto-Malay tribe, indiscriminate defecation, outdoor sewage disposal, eating contaminated fresh fruits, belonging to a large household members and earning low household income were significantly associated with the A. lumbricoides infection.

As for the hookworm infection, consuming raw vegetables was found to be significantly associated in the Negrito tribe. Moreover, univariate analysis identified only one factor that significantly associated with the infection among Senois which was eating contaminated fresh fruits. Among the Proto-Malay tribe, indiscriminate defecation, consuming raw vegetables, belonging to a large household members and earning low household income were significant risk factors for hookworm infection.

Risk factors for T. trichiura, A. lumbricoides and hookworm infections. Table 4 shows the results of the multivariate logistic regression analysis between $T$. trichiura, A. lumbricoides and hookworm infections and risk factors among the Proto-Malay, Negrito and Senoi tribes. The data confirmed consuming raw vegetables, belonging to a large household member and earning low household income as significant risk factors of T. trichiura infection in

Table 1 | General characteristics of the Orang Asli communities that participated in this study

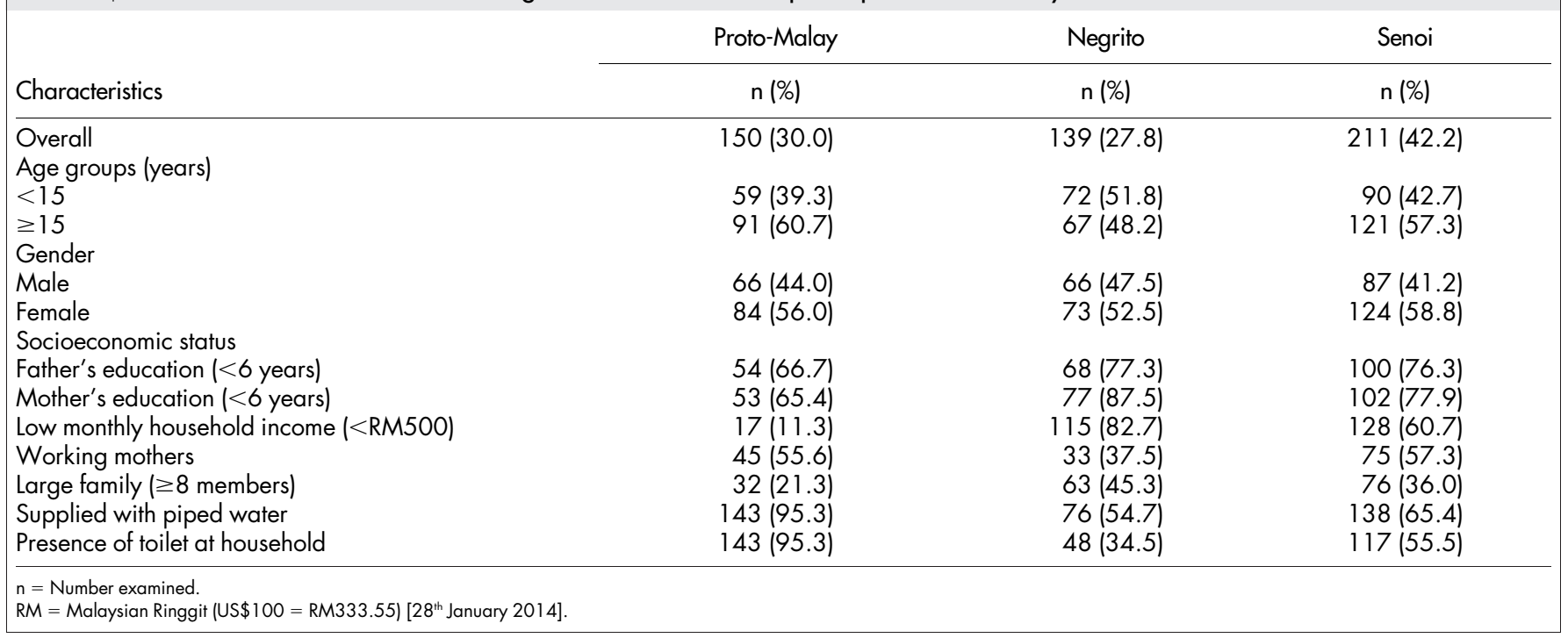


Table 2 | Prevalence of soil-transmitted helminths among Orang Asli according to age groups, gender and tribes

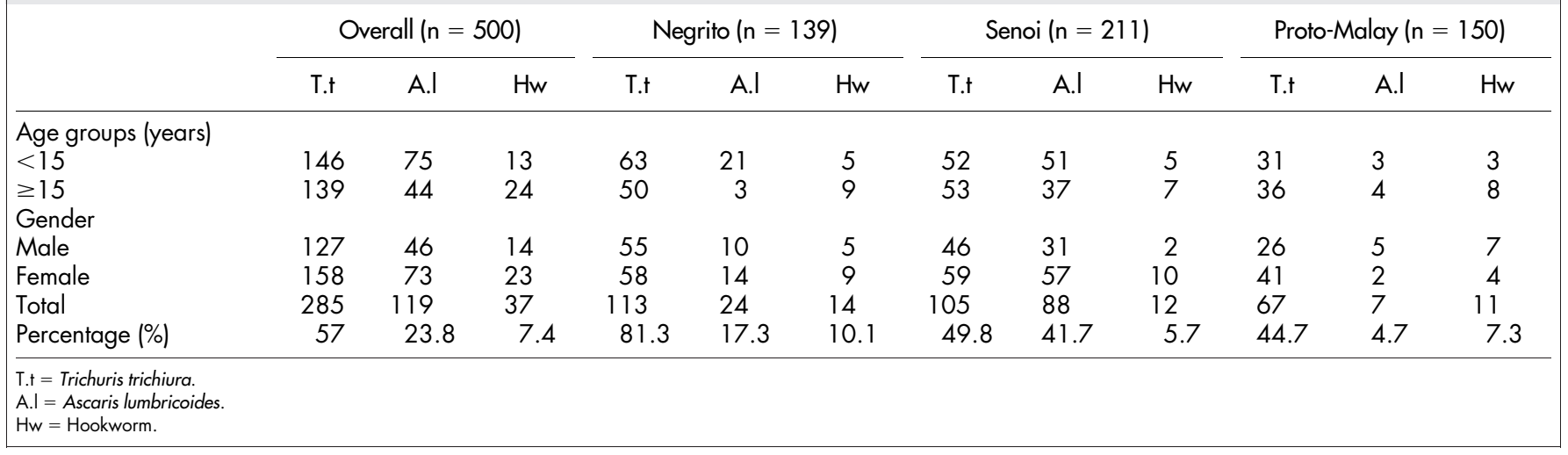

the Proto-Malay tribe. Furthermore, earning low household income and being aged $<15$ years were significant predictors identified in the Negrito and Senoi tribes, respectively. It also further confirmed that being aged less than 15 years was significant risk factor of ascariasis among the Negritos and Senois, whereas earning low household income was found to be a significant predictor among ProtoMalays. As for hookworm infection, consuming raw vegetables was found to be significant risk factor among the Proto-Malay and
Negrito tribes. Meanwhile, eating contaminated fresh fruits was significant predictor among the Senoi tribe.

Association of soil-transmitted helminth infections with diarrhoea and other gastrointestinal symptoms. There were no significant association between T. trichiura, A. lumbricoides and hookworm infections with diarrhoea or other gastrointestinal symptoms such as abdominal discomfort, vomiting and nausea in all tribes studied.

Table 3 | Univariate analysis of potential risk factors associated with soil-transmitted helminth infections among different Orang Asli tribes Species/Tribe/Variable Odds Ratio $95 \% \mathrm{Cl}$

$P$-value

Trichuris trichiura

(a) Proto-Malay

Indiscriminate defecation

1.12

1.50

Consuming raw vegetables

Low mother's education ( $<6$ years)

Large household members $(\geq 8)$

Low household income ( $<$ RM500)

(b) Negrito

Being aged less than 15 years

Bathing and washing in the river

Low father's education ( $<6$ years)

Low household income ( $<$ RM500)

(c) Senoi

Being aged less than 15 years

Ascaris lumbricoides

(a) Proto-Malay

Indiscriminate defecation

Outdoor sewage disposal

Eating contaminated fresh fruits

Large household members $(\geq 8)$

Low household income ( $<$ RM500)

(b) Negrito

Being aged less than 15 years

(c) Senoi

Being aged less than 15 years

Hookworm

(a) Proto-Malay

Indiscriminate defecation

Consuming raw vegetables

Large household members $(\geq 8)$

Low household income ( $<$ RM500)

(b) Negrito

Consuming raw vegetables

(c) Senoi

Eating contaminated fresh fruit

$\mathrm{Cl}=$ Confidence Interval.

$\mathrm{RM}=$ Malaysian Ringgit (US $\$ 100=\mathrm{RM} 333.55)$ [28 $8^{\text {th }}$ January 2014].

Significant association $(P<0.05)$.
2.65

1.25

1.18

1.48

1.74

2.71

3.10

1.31

1.71

1.53

1.53

1.88

1.58

4.45

1.59

1.54

3.36

1.48

1.42

3.42

5.19
$1.02,1.23$

$1.11,2.02$

$1.27,5.50$

$1.05,1.50$

$1.04,1.33$

$1.04,2.09$

$1.24,2.45$

$1.30,5.68$

$1.56,6.19$

$1.04,1.67$

$1.80,3.13$

$1.80,2.91$

$1.70,2.81$

$1.80,4.43$

$1.83,3.01$

$1.53,12.99$

$1.21,2.09$

$1.10,2.51$

$1.95,11.86$

$1.56,2.55$

$1.91,2.23$

$1.35,12.48$

$1.79,34.10$
0.007

0.005

0.004

0.009

0.006

0.050

0.007

0.015

0.002

0.022

$<0.001$

0.024

0.014

0.018

0.007

$<0.001$

$<0.001$

$<0.001$

0.005

0.042

0.007

0.014

0.017 
Table 4 | Multivariate analysis of risk factors associated with soil-transmitted helminth infections among the Proto-Malay, Negrito and Senoi tribes

Trichuris trichiura

(a) Proto-Malay

Consuming raw vegetables

Large household members $(\geq 8)$

Low household income ( $<$ RM500)

(b) Negrito

Low household income

(c) Senoi

Being aged less than 15 years

Ascaris lumbricoides

(a) Proto-Malay

Low household income

(b) Negrito

Being aged less than 15 years

(c) Senoi

Being aged less than 15 years

Hookworm

(a) Proto-Malay

Consuming raw vegetables

(b) Negrito

Consuming raw vegetables

(c) Senoi

Eating contaminated fresh fruit

$\mathrm{Cl}=$ Confidence Interval.

$R M=$ Malaysian Ringgit $($ US $\$ 100=R M 333.55)\left[28^{\text {th }}\right.$ January 2014]

Significant association $(P<0.05)$.

\section{Discussion}

It is evident from our results that soil-transmitted helminth (STH) infections are still prevalent and constitute a major public health problem among Orang Asli communities in Malaysia. The overall prevalence rates of STHs were in agreement with those of local studies where trichuriasis was found to be the most prevalent (range from $26 \%$ to $98.2 \%$ ), followed by ascariasis (range from $19 \%$ to $67 \%$ ) and lastly hookworm infection (range from $3 \%$ to $37 \%)^{20-22}$. An earlier study of Orang Asli in Selangor reported that the overall prevalence of ascariasis, trichuriasis and hookworm infection were $62.9 \%, 91.7 \%$ and $28.8 \%$, respectively ${ }^{10}$. However, observations in other parts of the world have recorded that $A$. lumbricoides and hookworm infections are more common than T. trichiura infection ${ }^{23-26}$. The higher prevalence rate of trichuriasis was also observed in previous studies ${ }^{10,12,27}$ and reported to be due to potential resistance of T. trichiura to most of anthelminthic drugs ${ }^{11,18}$.

It is interesting to note that although the distribution of STH infections according to species has remained the same (high prevalence of T. trichiura) for the last decades, the overall prevalence rates of STH infections in the present study have decreased significantly. The prevalence rate of $T$. trichiura has decreased from $67 \%-98 \%$ to 57\%; similar observations were also made in A. lumbricoides and hookworm infections where the prevalence rates have dropped to $23.8 \%$ from $51 \%$ and $7.4 \%$ from $23.5 \%$, respectively ${ }^{10,13,28}$. Meanwhile, trichuriasis and ascariasis were significantly high in the Negrito and Senoi tribes. This study also noted low STH infections in the Proto-Malay tribe.

In Malaysia, most of the Proto-Malay tribe used to migrate often but this practice has decreased over the past ten years. With better understanding of health and more permanent residence and better health care, the prevalence of STH has decreased from as high as $91.3 \%$ as reported by Dunn ${ }^{29}$ to $59.5 \%$ in the current study. On the other hand, most of the Negrito and Senoi tribes still pursue nomadic lifestyles such as hunting, fishing, gathering fruits and herbs for sale or part-time cultivators. With the exception of the study conducted by Dunn $^{29}$, most studies failed to focus on the tribe, culture and environment of the Orang Asli. Therefore, the current study found the importance of studying these particular groups as they live in diverse ecosystems and have diverse cultural practices and different approached to manage illness.

In Senois, being aged $<15$ years was identified as a predictor of trichuriasis. Meanwhile, ascariasis was significantly high among those aged less than 15 years in both the Negrito and Senoi tribes. This is in line with previous findings and epidemiology of STH infections which in such that children beginning starts to explore the environment get affected most ${ }^{12,13}$. It has also been reported that high prevalence rates persist after infancy through to adulthood with heavy worm burdens are found mainly among children ${ }^{30,31}$. A study done by Noor Azian et al. ${ }^{32}$ revealed that the highest infection rate was found in children aged less than 10 years old compared to other age groups even though it was not significantly different. During the visits to the villages, we observed that toddlers and young children were playing in groups outside the houses barefoot and eating unwashed vegetables and fruits contaminated with soil. Furthermore, children are more likely than adults to defecate in the open environment and to play in areas where excreta are dispersed, either by open defecation or by discarding latrine contents. Consistent with typical distribution patterns, hookworm prevalence was highest among young adults, although the reasons for this pattern remain unclear $^{33,34}$. Therefore, chemotherapy targeting children aged $<15$ years is an appropriate strategy for controlling T. trichiura and A. lumbricoides infections. Several studies have reported a higher prevalence of infection in males than females ${ }^{35,36}$ whereas other studies have indicated the opposite finding ${ }^{37,38}$. In the current study, the overall prevalence rates were not different between females and males. It is because both genders have an equal exposure to get these infections.

The prevalence rates of STH infections are also closely related to socioeconomic and overcrowded conditions. In this study, more than $60 \%$ of the population in Negritos and Senois villages were categorised under low household incomes family (<RM500), the poverty income threshold in Malaysia ${ }^{39}$. This leads to poor housing 
conditions and lacking of basic amenities as observed in villages of the Negrito and Senoi tribes which are difficult to maintain a good level of personal hygiene in this condition, resulting in favourable places for development of STHs ova in the soil and transmission of STH infections in these communities. This finding was also in agreement with previous studies conducted by other researchers ${ }^{35,40}$. Osten et al. ${ }^{41}$ and Maia et al. ${ }^{42}$ reported that increased number of household members and overcrowded conditions are associated with higher frequency of parasitic infections. Our results concur with these findings. Presence of eight members or more in the family increased the risk of infection which may be attributed to the close contact within the crowded houses and lead to increase risk of intra-family transmission. This is in contradiction to the work of Quihui et $\mathrm{al}^{35}$ which observed that family size had no significant effect on the rate of parasitic infection.

Other important factors contributing to the T. trichiuris and hookworm infections in the Proto-Malay and Senoi tribes were activities related to poor food hygiene such as consuming raw vegetables and eating contaminated fresh fruits. Soil-transmitted helminths are significantly associated with environmental conditions such as the contamination of soil with human faeces ${ }^{43}$ and poor sewage disposal including the use of night soil as fertiliser ${ }^{44}$. When soil becomes contaminated, helminth eggs in the soil can be transferred to vegetables, then on to hands and then directly in the mouth ${ }^{45}$ or ingested by eating raw vegetables or fresh fruits ${ }^{44}$. Steinmann ${ }^{46}$ has reported that washing raw vegetables before eating them was protective against STHs. Thus, health education related to food hygiene is the main strategies in the control of trichuriasis and hookworm infection in both tribes.

We acknowledge several limitations of our methodology. The formalin-ether sedimentation and Wheatley's trichrome staining techniques employed for processing faecal samples do not yield data on parasite intensity but only on the prevalence rate. The methods were chosen for their efficiency and cost-effectiveness but are less sensitive than other commonly used procedures. Additional technique such as Kato-Katz would have been able to provide useful data on the relative intensity of infections. Harada-Mori culture was not carried out due to the limitation of the faecal samples. Therefore, the hookworm species could not be detected in this study. However in Malaysia, hookworm infection is caused predominantly by Necator americanus $^{29}$.

The present study provides reasonably good estimates of prevalence for most species but not, of course for Enterobius vermicularis or Strongyloides stercoralis. Pinworm surveys were not attempted. The culture of $S$. stercoralis was not feasible under field conditions; although it would have been possible in the hospital surveys, it was omitted for lack of time. The prevalence of E. vermicularis could not be estimated as the refusal rate for the application of the cellophane tape was high.

Only one faecal sample was examined instead of the ideal three consecutive samples due to the level of cooperation and response of the parents and guardians. Therefore, there is likely to be a significant underestimation of infection. This underestimation is based on the fact that a single sample misses infection in an individual because of the temporal variation in egg excretion over hours and days. It has been shown that obtaining only a single sample for A. lumbricoides and T. trichiura can underestimate infection rates by up to $50 \%{ }^{47}$.

Finally, the current study did not offer a quantify look at the physical effects of STH infections. Children infected with STHs are expected to have significantly lower weight-for-age and height-forage $\mathrm{z}$-scores than do healthy children, putting them at risk for a number of conditions associated with malnutrition.

The present study demonstrated significantly high prevalence rates of $T$. trichiura and $A$. lumbricoides infections among the Negrito and Senoi tribes. This study also identified several factors that contribute to the difference in infection prevalence between the three tribes. Given that STHs are intimately associated with poverty, poor hygienic practices and lack of proper basic amenities, it is crucial that these factors are addressed effectively. Different preventive programs are required including health education and promotion, awareness about personal hygiene and environmental sanitation. In addition, scheduled deworming targeting children aged less than 15 years with proper monitoring of the program has to be implemented to help in reducing the community worm burden and re-infection rate. Last but not least, future study may be conducted using more comprehensive diagnostic techniques to determine not only the prevalence of infection but also the intensity and species.

\section{Methods}

Study areas. The present study was carried out from June to December 2011 in three different states of Peninsular Malaysia without discriminating age or gender. Specific villages within the locations of Jelebu $\left(2^{\circ} 55^{\prime} \mathrm{N}\right.$ latitude, $102^{\circ} 4^{\prime}$ E longitude), Gerik $\left(5^{\circ} 26^{\prime} \mathrm{N}\right.$ latitude, $101^{\circ} 7^{\prime} \mathrm{E}$ longitude) and Temerloh $\left(3^{\circ} 43^{\prime} \mathrm{N}\right.$ latitude, $102^{\circ} 22^{\prime} \mathrm{E}$ longitude) in Malaysia were included in this study. These three states have a similar climate; which is a tropical rainforest climate, being hot and humid throughout the year. Orang Asli is a collective term for a group of indigenous people that usually reside in the interior regions of Peninsular Malaysia. They identify themselves by tribes i.e., Proto-Malay, Negrito and Senoi. They comprise about $0.6 \%$ of the total population in Malaysia.

Parit Gong village, Jelebu, Negeri Sembilan state is considered a sub-urban area with a population of 496 inhabitants. Most of the residents are primarily involved in farming and rubber tapping. There are also many individuals who are engaged in commercial and professional activities such as teaching and government service ${ }^{48}$. The average temperature in this area is $25.9^{\circ} \mathrm{C}$ with an average rainfall of $142 \mathrm{~mm} /$ year ${ }^{49}$. The main sub-ethnic group residing in this area is the Temuan and who belong to the Proto-Malay tribe.

RPS Air Banun area, Gerik, Perak state is considered a remote area, located in a valley approximately 40 kilometres from the town of Gerik. The occupations of the inhabitants of the town include farming, rubber tapping and some do odd jobs such as selling forest products. The area comprises of five villages with 10 to 15 households in $e^{e a c h}{ }^{50}$. The average temperature in this area is $26.9^{\circ} \mathrm{C}$ with an average rainfall of $180 \mathrm{~mm} /$ year $^{49}$. The main sub-ethnic group residing in this area is the Jahai who belongs to the Negrito tribe.

Pasu village, Temerloh, Pahang state is considered a suburban area which is within 10 kilometres from the town of Kuala Krau. Out of 625 residents, $65 \%$ are farmers and rubber tappers while the remainders are mostly government and private workers ${ }^{51}$. The average temperature in this area is $26.6^{\circ} \mathrm{C}$ with an average rainfall of $161 \mathrm{~mm} /$ year $^{49}$. The main sub-ethnic group residing in this area is the Jahut and who belong to the Senoi tribe.

Sample size and sampling strategy. The sample size was calculated for the primary objective taking the prevalence to be estimated at $50 \%$ for gives the maximum sample size, with $95 \%$ level of confidence and $5 \%$ bound on the error of estimation. The minimum sample size required was 385 participants. Simple random sampling method was used to select the households. A list of all the households (the sampling frame) was prepared in a preliminary census type survey.

Questionnaire survey. A structured questionnaire was developed in English and then translated to Malay language (the national language for Malaysia). The questionnaire was pre-tested among Orang Asli who was admitted to Gombak Hospital, Selangor state. Trained research assistants interviewed participants in person, asking questions on demographic data (i.e. age, gender and education level), socioeconomic background (i.e. occupation, household income and educational status), behavioural risks (i.e. personal hygiene such as hand washing and food consumption), environmental sanitation and living condition characteristics (i.e. types of water supply, latrine system, sewage disposal system and presence of domestic animals) and health conditions with history of symptoms (i.e. diarrhoea, nausea, vomiting and abdominal pain). For children ( $\leq 12$ years old), answers from the questionnaire were double-confirmed by interviewing their parents or the guardian who had given informed consent.

Faecal sample collection and analysis. Following the administration of the questionnaire, a wide mouth $100 \mathrm{~mL}$ screw-capped container pre-labelled with the individual's name and code was distributed to each participant for the collection of a faecal sample the next day. Their ability to recognize their name was counter-checked. The participant was instructed to scoop a thumb sized faecal sample using a provided scoop into the container. Then, the container was placed into a zip-locked plastic bag. Parents and guardians were instructed to monitor their children during the sample collection in order to ensure that they placed their faecal samples into the correct container. All study participants were asked to provide sufficiently large faecal sample (at least 10 grams) so that both formalin-ether sedimentation and Wheatley's trichrome staining techniques could be performed. This study had to rely on a single faecal sample collection. It was not feasible to collect three faecal samples due to the cultural belief of the Orang Asli against giving of their faecal samples. However, we 
have applied standard procedures during faecal collection and examination to overcome this limitation.

Faecal samples were processed in the designated area of work in the study village within a maximum of four hours after collection by experienced laboratory technicians. Approximately 5 grams of each faecal sample was kept into a $15 \mathrm{~mL}$ centrifuge tube containing $3 \mathrm{~mL}$ polyvinyl alcohol (PVA). PVA-fixed samples were forwarded to the parasitological department of the Faculty of Medicine, Universiti Kebangsaan Malaysia. The samples were subjected to Wheatley's trichrome staining. Briefly, the smear cover slip was stained as follows: iodine alcohol (15 minutes), 70\% alcohol (10 minutes), trichrome stain (10 minutes), acid alcohol (3 seconds), 95\% alcohol (5 minutes), absolute alcohol (5 minutes) and winter green oil $(5 \text { minutes })^{52}$. The cover slip was mounted using DPX and examined under light microscope at magnifications of $\times 100$. Additionally, another half of the samples were kept unfixed and stored at $4{ }^{\circ} \mathrm{C}$ upon arrival at the laboratory for further analysis using formalin-ether sedimentation. Briefly, 2 grams of faecal sample was mixed with $7 \mathrm{~mL}$ of formalin and $3 \mathrm{~mL}$ of ether, centrifuged, stained with Lugol's iodine and finally examined under light microscope at magnifications of $\times 40^{53}$. Sample was reported as positive if ova of helminths were detected by any of the two techniques.

Statistical analysis. Statistical analysis was performed using the SPSS version 20 (SPSS, Chicago, IL, USA). Data was entered in a Microsoft Access and was crosschecked by the technical staff in order to ensure that data were entered correctly. Before each analysis, data were again checked for consistency. Only those individuals who had formalin-ether sedimentation and Wheatley's trichrome staining results together with complete questionnaire data were included in the final analyses.

For descriptive analysis, rate (percentage) was used to describe the characteristics of the studied population, including the prevalence of STHs. A Chi-squares test $\left(\chi^{2}\right)$ was used to test the associations between the variables. In the univariate analysis, the dependent variable was prevalence of STHs, while the independent variables were demographic and socioeconomic factors, behavioural risks, environmental sanitation, living condition characteristics, and gastrointestinal symptoms. All variables that were significantly associated with the prevalence of STHs in the univariate model were included in a logistic regression analysis to identify the risk factors for STHs. For each statistically significant factor, an Odds Ratio (OR) and 95\% confidence interval (CI) were computed by the univariate and multivariate logistic regression analyses. The level of statistical significance was set as $P<0.05$ because of the small number of participants $(\mathrm{n}=500)$.

Ethical considerations and treatment. Prior to data collection, the study protocol (Reference Number: UKM 1.5.3.5/244/FF-165-2011) was reviewed and approved by the Ethics Committee of Universiti Kebangsaan Malaysia Medical Centre (UKMMC) and permission for field work was obtained from Department of Orang Asli Development (JAKOA). Village meetings were held and village authorities and villagers were handed detailed explanations of the aims, procedures, potential risks and benefits of the study. During the meeting, they were also informed that their identity and personal information would be kept strictly confidential, and they could withdraw from the study at any point of time without citing reasons for doing so. If they agreed to participate, their consent was obtained in written form (signature) or thumbprint (for those who were illiterate). The parents or guardians were approached for written informed consent on behalf of their children ( $\leq 12$ years old) to give their faecal samples. According to Malaysian law, the parents or guardians need to sign on behalf of their children under 18 years old. All participants that were diagnosed positive with STHs were treated accordingly by the medical personnel from the Ministry of Health, Malaysia at no cost to them.

1. Hotez, P. J. et al. Control of neglected tropical diseases. N Engl J Med. 357, 1018-1027 (2007).

2. Hotez, P. J. One world health: Neglected Tropical Diseases in a Flat World. PLoS Negl Trop Dis. 3, e405 (2009).

3. Liese, B., Rosenberg, M. \& Schratz, A. Programmes, partnerships and governance for elimination and control of neglected tropical diseases. Lancet. 375, 67-76 (2010).

4. Vandemark, L. M., Jia, T. W. \& Zhou, X. N. Social science implications for control of helminth infections in Southeast Asia. Adv Parasitol. 73, 137-170 (2010).

5. Alemu, A. et al. Soil-transmitted helminths and Schistosoma mansoni infections among school children in Zarima town, northwest Ethiopia. BMC Infect Dis. 11, 189 (2011).

6. Bethony, J. et al. Soil-transmitted helminth infections: ascariasis, trichuriasis and hookworm. Lancet. 367, 1521-1532 (2006).

7. Holland, C. V. Predisposition to ascariasis: patterns, mechanisms and implications. Parasitology. 136, 1537-1547 (2009).

8. WHO. Deworming for health and development. Report of the third global meeting of the Partners for Parasite Control. Geneva. World Health Organization (2005).

9. King, C. H. Parasites and poverty: the case of schistosomiasis. Acta Trop. 113, 95-104 (2010).
10. Norhayati, M. et al. The prevalence of Trichuris, Ascaris and hookworm infection in Orang Asli children. Southeast Asian J Trop Med Public Health. 28, 161-167 (1997).

11. Norhayati, M., Peggabean, M., Oothuman, P. \& Fatmah, M. S. Prevalence and some risk factors of Giardia duodenalis infection in rural communities in Malaysia. Southeast Asian J Trop Med Public Health. 29, 735-738 (1998).

12. Al-Mekhlafi, M. S. et al. Prevalence and distribution of soil-transmitted helminthiases among Orang Asli children living in peripheral Selangor, Malaysia. Southeast Asian J Trop Med Public Health. 37, 41-47 (2006).

13. Al-Mekhlafi, M. S. et al. Pattern and predictors of soil-transmitted helminth reinfection among aboriginal school children in rural Peninsular Malaysia. Acta Trop. 107, 200-204 (2008).

14. Rahmah, N. et al. Parasitic infections among Aborigine children at Post Brooke, Kelantan. Med J Malaysia. 52, 412-414 (1997).

15. Rahman, W. A. The prevalence and intensity of soil-transmitted helminthes in some rural villages in northern peninsular Malaysia. Southeast Asian J Trop Med Public Health. 25, 296-299 (1994).

16. Oothuman, P. et al. The prevalence and reinfection of intestinal helminthiases among school children in an estate. Trop Biomed. 12, 159-164 (1995).

17. Chan, L., Kan, S. P. \& Bundy, D. A. P. The effect of repeated chemotherapy on the prevalence and intensity of Ascaris lumbricoides and Trichuris trichiura infection. Southeast Asian J Trop Med Public Health. 23, 228-233 (1992).

18. Rajeswary, B., Sinniah, B. \& Hasnah, H. Socioeconomic factors associated with intestinal parasites among children living in Gombak, Malaysia. Asia Pac J Public Health. 7, 21-25 (1994)

19. Rahman, W. A. Helminthic infections of urban and rural schoolchildren in Penang Island, Malaysia. Southeast Asian J Trop Med Public Health. 29, 596-598 (1998).

20. Nor Aini, U. et al. Serum iron status in Orang Asli children living in endemic areas of soil-transmitted helminthes. Asia Pac J Clin Nutr. 16, 724-730 (2007).

21. Kan, S. P. \& Poon, G. K. Prevalence, distribution and intensity of soil-transmitted helminthiases among Malaysian children. Public Health. 101, 243-251 (1987).

22. Hanjeet, K., Lai, K. P. F., Mathias, R. G. \& OwYang, C. K. Soil-transmitted helminthiases in squatter populations around Kuala Lumpur by ethic distribution. Trop Biomed. 8, 33-37 (1991).

23. World Health Organization. Prevention and control of intestinal parasitic infections. Geneva (1987) (http://whqlibdoc.who.int/trs/WHO_TRS_749.pdf). Accessed: 3 May 2013.

24. Kang, G. et al. Prevalence of intestinal parasites in rural Southern Indians. Trop Med Int Health. 3, 70-75 (1998).

25. Brooker, S. et al. The epidemiology of hookworm infection and its contribution to anemia among pre-school children on the Kenya coast. Trans $R$ Scop Trop Med Hyg. 93, 240-246 (1999).

26. Lwambo, N. J. et al. Pattern and concurrent hookworm infection and schistosomiasis in schoolchildren in Tanzania. Trans R Soc Trop Med Hyg. 93, 497-502 (1999).

27. Sinniah, B. et al. Determining the prevalence of intestinal parasites in three Orang Asli (Aborigines) communities in Perak, Malaysia. Trop Biomed. 29, 200-206 (2012).

28. Ngui, R. et al. Prevalence and risk factors of intestinal parasitism in rural and remote west Malaysia. PLoS Negl Trop Dis. 5, e974 (2011).

29. Dunn, F. L. Intestinal parasites in Malayan aborigines (Orang Asli). Bull World Health Organ. 46, 99-113 (1972).

30. Elkins, D. B., Haswell-Elkins, M. \& Anderson, R. M. The epidemiology and control of intestinal helminthes in the public at lake region of southern India. Study design and pre and post-treatment observation on Ascaris lumbricoides infection. Trans R Soc Trop Med Hyg. 80, 744-792 (1986).

31. Bundy, D. A. P. et al. Age related changes in the prevalent and intensity of Trichuris trichiura in a St. Lucia community. Trans $R$ Soc Trop Med Hyg. 81, 85-89 (1987)

32. Noor Azian, M. Y. et al. Prevalence of intestinal protozoa in an aborigine community in Pahang, Malaysia. Trop Biomed. 24, 55-62 (2007).

33. Anderson, T. J. C. et al. The distribution of intestinal helminth infections in a rural village in Guantemala. Mem Inst Oswaldo Cruz. 88, 53-65 (1993).

34. Hotez, P. J. et al. Hookworm infection (review article). N Engl J Med. 351, 799-807 (2004).

35. Quihui, L. et al. Role of the employment status and education of mothers in the prevalence of intestinal parasitic infections in Mexican rural schoolchildren. BMC Public Health. 6, 225-232 (2006).

36. Nasiri, V. et al. Intestinal parasitic infections among ingabitants of Karaj city, Tehran province, Iran in 2006-2008. Korean J Parasitol. 47, 265-268 (2009).

37. Sharif, M., Daryani, A., Asgarian, F. \& Nasrolahei, M. Intestinal parasitic infections among intellectual disability children in rehabilitation centers of northern Iran. Res Dev Disabil. 31, 924-928 (2010).

38. Warunee, N. et al. Intestinal parasitic infections among school children in Thailand. Trop Biomed. 24, 83-88 (2007).

39. Department of Statistics Malaysia. Profile of Orang Asli in Peninsular Malaysia, Kuala Lumpur (http://www.statistics.gov.my). Accessed: 3 May 2013.

40. Nematian, J., Nematian, E., Gholamrezanezhad, A. \& Asgari, A. A. Prevalence of intestinal parasitic infections and their relation with socio-economic factors and hygienic habits in Tehran primary school students. Acta Trop. 92, 179-186 (2009). 
41. Ostan, I. et al. Health inequities: lower socio-economic conditions and higher incidences of intestinal parasites. BMC Public Health. 7, 342-349 (2007).

42. Maia, M. M. et al. Intestinal parasitic infections and associated risk factors among children presenting at outpatient clinics in Manaus, Amazonas state, Brazil. Ann Trop Med Parasitol. 103, 583-591 (2009).

43. Muttalib, M. A., Huq, M., Huq, J. A. \& Suzuki, N. Soil pollution with Ascaris ova in three villages of Bangladesh. 66-71 (Asian Parasite Control Organization, Tokyo, 1983).

44. Mustafa, U. et al. Environmental pollution with soil-transmitted helminths in Sanliurfa, Turkey. Mem Inst Oswaldo Cruz. 96, 903-909 (2001).

45. Koyabashi, A. Ascariasis: Textbook for seminar on parasite control administration for senior officers - a step towards primary health care. 233-242. (International Cooperation Agency, Japan, 1999).

46. Steinmann, P. et al. Rapid appraisal of human intestinal helminth infections among schoolchildren in Osh oblast, Kyrgyzstan. Acta Trop. 116, 178-184 (2010).

47. Knopp, S. et al. Diagnosis of soil-transmitted helminths in the era of preventive chemotherapy: Effect of multiple stool sampling and use of different diagnostic techniques. PLoS Negl Trop Dis. 2, e331 (2008).

48. Department of Orang Asli Development (JAKOA) Research and Development Division: Information Database, Jelebu District, Negeri Sembilan, Malaysia (http://www.jakoa.gov.my). Accessed: 3 May 2013.

49. Malaysia Meteorological Department. Ministry of Science, Technology and Innovation Database, Malaysia (http://www.met.gov.my). Accessed: 3 May 2013.

50. Department of Orang Asli Development (JAKOA) Research and Development Division: Information Database, Gerik District, Perak, Malaysia (http://www. jakoa.gov.my). Accessed: 3 May 2013.

51. Department of Orang Asli Development (JAKOA) Research and Development Division: Information Database, Temerloh District, Pahang, Malaysia (http:// www.jakoa.gov.my). Accessed: 3 May 2013.

52. Salleh, F. M., Anuar, T. S., Yasin, A. M. \& Moktar, N. Wintergreen oil: A nove method in Wheatley's trichrome staining technique. J Microbiol Methods. 91, 174-178 (2012).
53. Fleck, S. L. \& Moody, A. H. Diagnostic technique in medical parasitology. 10-14 (Cambridge University Press, Cambridge, 1993).

\section{Acknowledgments}

The authors are very grateful to the Department of Orang Asli Development (JAKOA), Ministry of Rural and Regional Development, Kuala Lumpur, Malaysia for granting us permission to conduct this research. Special thanks also go to all participants from Parit Gong village, Pasu village, and RPS Air Banun for their commitment and contribution in providing their faecal samples. The work presented in this paper was funded by UKMMC Fundamental Research Grant (FF-165-2011) and Special Research University Grant (UKM-GUP-2011-316) from Universiti Kebangsaan Malaysia.

\section{Author contributions}

T.S.A. and N.M. conceived, planned and designed experiments. T.S.A. and N.M. contributed reagents/materials/analysis tools. T.S.A. and F.M.S. conducted the experiments. T.S.A. and N.M. analyzed the data. T.S.A. and N.M. wrote the paper. All authors reviewed the manuscript.

\section{Additional information}

Competing financial interests: The authors declare no competing financial interests.

How to cite this article: Anuar, T.S., Salleh, F.M. \& Moktar, N. Soil-Transmitted Helminth Infections and Associated Risk Factors in Three Orang Asli Tribes in Peninsular Malaysia. Sci. Rep. 4, 4101; DOI:10.1038/srep04101 (2014).

\footnotetext{
(c) (i) (2) This work is licensed under a Creative Commons Attribution-

cc.
} visit http://creativecommons.org/licenses/by-nc-sa/3.0 